\title{
Perfil glicêmico de caminhoneiros que trafegam por uma capital do nordeste brasileiro
}

\author{
Glycemic profile of truckers through a capital of northeast Brazil \\ Perfil glicémico de camiones através de una capital del noreste de Brasil
}

Antônio Tiago da Silva Souza ${ }^{1 *}$, Anderson da Silva Sousa ${ }^{2}$, André Felipe Pereira Severo ${ }^{3}$, Andressa Carvalho Pereira ${ }^{1}$, Cindy Heloisa da Silva Viana ${ }^{2}$, Delmo de Carvalho Alencar ${ }^{3}$, Francisco Ricardo Nascimento Freitas ${ }^{1}$, Marianne dos Santos Pereira ${ }^{4}$, Renata Giordana de Sousa Andrelino ${ }^{2}$, Telma Maria Evangelista de Araújo $^{3}$.

\begin{abstract}
RESUMO
Objetivo: Avaliar o perfil glicêmico de caminhoneiros que trafegam por Teresina, Piauí. Métodos: Trata-se de estudo epidemiológico, transversal, mediante entrevistas e tomada de peso, altura, pressão arterial e glicemia capilar. Participaram do estudo 74 caminhoneiros. Os dados foram digitados e analisados com a utilização do software Statistical Package for the Social Sciences versão 17.0. Foram realizadas análises univariadas, por meio de estatísticas descritivas simples e bivariadas, com aplicação do teste Qui-quadrado de Pearson e test $t$ com nível de significância $(p<0,05)$. Resultados: $75,7 \%$ da população estudada encontrava-se com glicemia normal e 24,3\% apresentaram glicemia acima dos níveis considerados normais com máxima de $490 \mathrm{mg} / \mathrm{dL}$. Observou-se que a expressiva maioria dos caminhoneiros (69\%) tem uma alimentação inadequada, apenas $31 \%$ referiu consumo alimentar dentro do padrão considerado adequado pelo estudo. O índice de massa corpórea variou de 20 a 38, com média 28,4, demonstrando sobrepeso na população da pesquisa. Conclusão: Há necessidade de uma atenção voltada para a promoção e proteção específica da saúde destes trabalhadores, considerando-se as suas dificuldades de acesso aos serviços de saúde.
\end{abstract}

Palavras-chave: Saúde do homem, Diabetes mellitus, Epidemiologia.

\begin{abstract}
Objective: To evaluate the glycemic profile of truck drivers traveling through Teresina, Piauí. Methods: This is an epidemiological, cross-sectional study, using interviews and taking weight, height and capillary blood glucose. 74 truck drivers participated in this study. The data were entered and analyzed using the Statistical Package for the Social Sciences software version 17.0. Univariate analyzes were performed using simple and bivariate descriptive statistics, using Pearson's chi-square test and t test with significance level $(p<0.05)$. Results: $75.7 \%$ of the studied population had normal blood glucose and $24.3 \%$ had blood glucose above levels considered normal with a maximum of $490 \mathrm{mg} / \mathrm{dL}$. It was observed that the expressive majority of truck drivers $(69 \%)$ have an inadequate diet, only $31 \%$ reported food consumption within the standard considered adequate by the study. The body mass index ranged from 20 to 38 with an average of 28.4 demonstrating overweight in the research population. Conclusion: There is a need for attention focused on the promotion and specific protection of the health of these workers, considering their difficulties in accessing health services.
\end{abstract}

Keywords: Men's health, Diabetes mellitus, Epidemiology.

\section{RESUMEN}

Objetivo: Evaluar el perfil glucémico de los camioneros que viajan por Teresina, Piauí. Métodos: Este es un estudio epidemiológico, transversal, que utiliza entrevistas y toma peso, altura y glucosa en sangre capilar.

\footnotetext{
1 Universidade Federal do Delta do Parnaíba (UFDPAR), Parnaíba - PI. *E-mail: at.tiago@ hotmail.com

2 Centro Universitário de Saúde, Ciências Humanas e Tecnológicas do Piauí (UNINOVAFAPI), Teresina - PI.

${ }^{3}$ Universidade Federal do Piauí (UFPI), Teresina - PI.

${ }^{4}$ Cristo Faculdade do Piauí (CHRISFAPI), Piripiri - PI.
} 
74 camioneros participaron en este estudio. Los datos se ingresaron y analizaron utilizando el software Statistical Package for the Social Sciences versión 17.0. Los análisis univariados se realizaron utilizando estadísticas descriptivas simples y bivariadas, utilizando la prueba de chi-cuadrado de Pearson y la prueba $t$ con nivel de significancia ( $p<0.05$ ). Resultados: $75.7 \%$ de la población estudiada tenía glucosa en sangre normal y $24.3 \%$ tenía glucosa en sangre por encima de los niveles considerados normales con un máximo de $490 \mathrm{mg} / \mathrm{dL}$. Se observó que la mayoría expresiva de los camioneros (69\%) tiene una dieta inadecuada, solo el 31\% informó el consumo de alimentos dentro del estándar considerado adecuado por el estudio. El índice de masa corporal varió de 20 a 38, con un promedio de 28.4 que demuestra sobrepeso en la población de investigación. Conclusión: Existe una necesidad de atención enfocada en la promoción y protección específica de la salud de estos trabajadores, considerando sus dificultades para acceder a los servicios de salud.

Palabras clave: Salud del hombre, Diabetes mellitus, Epidemiología.

\section{INTRODUÇÃO}

O diabetes mellitus (DM) é uma síndrome de etiologia múltipla, causada pela falta e/ou inadequados efeitos de insulina no organismo. Tem como característica a hiperglicemia crônica associada a distúrbios metabólicos dos carboidratos, lipídeos e proteínas. As consequências do DM, a longo prazo, incluem disfunção e falência de vários órgãos, especialmente rins, olhos, nervos e vasos sanguíneos (MALHEIROS TM, et al., 2017).

O diabetes tipo 1 (DM1) trata-se de doença auto-imune, em que as células beta pancreáticas, produtoras de insulina, são destruídas. Pode surgir em qualquer idade, porém acomete frequentemente pessoas com menos de 35 anos. Os principais sintomas evidenciados no DM1 são: poliúria, fome frequente, sede constante, perda de peso, fraqueza, fadiga, nervosismo, mudanças de humor, náuseas e vômitos, podendo ainda ser assintomático. O diabetes tipo 2 (DM2) tem forte relação com o sedentarismo e obesidade, além de ter a hereditariedade como grande fator de risco. Os sintomas de DM2 frequentemente não se manifestam ou são vagos, como formigamento nas mãos e pés, infecções frequentes, alteração visual (visão embaçada), dificuldade na cicatrização de feridas e furunculose. Portanto, é importante pesquisar diabetes em todas as pessoas com mais de 40 anos de idade, uma vez que as estimativas mostram que $60 \%$ a $90 \%$ dos portadores da doença, encontram-se nesta faixa etária e grande parte destes, desconhecem o diagnóstico da doença, causando subnotificação, diagnósticos tardios e aparecimento de complicações da doença (SMELTZER SC e BARE BG, 2015).

A doença cardiovascular aterosclerótica, neuropatia e retinopatia são complicações crônicas decorrentes do mau controle glicêmico. Para diagnosticar o diabetes é necessária a realização de alguns exames laboratoriais, onde os mais comumente realizados são: glicemia de jejum, teste oral de tolerância a glicose (TOTG-75g) e glicemia casual. A glicemia casual pode ser mensurada em qualquer momento do dia, sem considerar o tempo da última refeição. Os valores de referência para o diagnóstico de DM são: glicemia de jejum maior ou igual que $126 \mathrm{mg} / \mathrm{dL}$; glicemia plasmática após $2 \mathrm{~h}$ do TOTG maior ou igual que $200 \mathrm{mg} / \mathrm{dL}$; ou ainda glicemia casual igual ou superior a $200 \mathrm{mg} / \mathrm{dL}$ (DIAS ACM, et al., 2018).

O DM2 ocorre com frequência em pessoas acima de 40 anos de idade. Os principais fatores que contribuem para o surgimento do DM2 são etnia, genética, idade, sobrepeso, obesidade e sedentarismo, o que comprova que esse tipo de DM está fortemente relacionado à associação entre a predisposição genética e familiar e ao estilo de vida e fatores ambientais. Seu tratamento envolve alterações no estilo de vida, principalmente em relação aos hábitos alimentares, prática de atividade física e uso de medicamentos, que podem ser hipoglicemiantes orais ou insulina (ALENCAR DC, et al., 2019; BARROSO SV e BIAZON AZV, 2017). Uma alimentação descontrolada ou uma adesão inadequada à prescrição dietética incorre no mau controle do DM (SEIXAS AMFF, et al., 2016).

O DM é responsável em todo o mundo por mais de $14 \%$ dos óbitos por todas as causas. No Brasil, se configura como um dos principais agravos de saúde pública. De acordo com a International Diabetes Federation, o Brasil ocupava em 2015 a quarta posição no mundo no quantitativo de pessoas diabéticas, com 14,3 milhões de pessoas vivendo com o agravo. Segundo projeções, em 2040 este número aumentará para 23,3 milhões (FONTES FLL, et al., 2019). 
Dentre os grupos com prevalências preocupantes de fatores de risco para doenças crônicas, encontramse os caminhoneiros, considerando-se que na rotina de trabalho diário enfrentam curtos prazos de entrega e dificuldades para a realização de pausas de descanso, ocasionando em muitos destes, o consumo de substâncias químicas, como anfetaminas, na tentativa de se manterem acordados para atender os prazos determinados pelas empresas. Além disso sua atividade de trabalho exige grande carga horária, o que os leva a uma vida sedentária e a uma alimentação desfavorável, predispondo a várias doenças como diabetes mellitus, hipertensão, câncer, stress, doenças do sono, dentre outras (SILVA LG, et al., 2016).

Entendemos que as dificuldades para essa categoria profissional manterem uma alimentação saudável são grandes em função da constante ausência das suas casas, do próprio hábito alimentar adquirido ao longo da vida, da falta de tempo para a construção de uma educação em saúde, dentre outros aspectos. Desse modo, se por um lado é comum a observação de caminhoneiros em restaurantes de "beira de estrada" alimentando-se basicamente de massas, carnes vermelhas gordas, frituras em geral e açúcares, por outro, têm sido escassas as experiências de vê-los se alimentando de frutas, verduras e outros alimentos favoráveis à saúde. Esta prática alimentar aumenta a probabilidade do desenvolvimento de diabetes e das altas taxas glicêmicas, independentemente do índice de massa corpórea (IMC), grau de atividade física, idade ou história familiar do paciente (GIROTTO E, et al., 2020; DIAS SM, et al., 2018).

Diante do exposto, o estudo teve como objetivo avaliar o perfil glicêmico de caminhoneiros que trafegam por Teresina, Piauí, Brasil.

\section{MÉTODOS}

O presente estudo é de natureza quantitativa, descritiva, seccional e foi desenvolvido no município de Teresina, capital do estado do Piauí, cuja população de acordo com o Instituto Brasileiro de Geografia e Estatísticas (IBGE), é de 864.845 habitantes (IBGE, 2019). O local escolhido para coleta dos dados foi um posto de combustíveis localizado na zona sul da cidade, onde a expressiva maioria dos caminhoneiros que trafegam pela capital escolhem para o repouso e pernoites. O período da coleta de dados foi entre os meses de maio e junho de 2014.

A população do estudo foi composta pelos caminhoneiros que trafegavam por Teresina. Com relação ao seu tamanho foram considerados estudos sobre a prevalência de diabetes na população adulta, acima de 18 anos, cujo valor foi de 6,2\% (ISER BPM, et al., 2015). Outro estudo utilizado como referência, aferiu a prevalência de DM no Brasil, a partir de um survey nacional, que indicou prevalência de DM de 7,5\%, apontando a necessidade de mudanças comportamentais como estratégia para prevenção e controle do diabetes e suas complicações (FLOR LS e CAMPOS MR, 2017). Assim, adotou-se uma prevalência presumida de $5 \%$, com erro tolerável de amostragem de $5 \%$ e um nível de confiança de $95 \%$, sendo adotada a fórmula a seguir: $n=Z^{2}$. (p.q) $/ e^{2}$. Onde $n=$ tamanho da população; $Z=$ intervalo de confiança; $p=$ prevalência presumida; $q=1-p ; e=$ erro tolerável.

A seleção da população foi por amostragem acidental, a qual se formou pelos caminhoneiros que foram aparecendo, até completar o número pretendido pela amostra (LUIZ RR, 2009). As variáveis estudadas foram: idade, sexo, renda per capita, estado civil, escolaridade, tempo de trabalho como caminhoneiro, peso, altura, pressão arterial, hábitos alimentares, glicemia casual e histórico familiar de diabetes.

Para a coleta de dados, utilizou-se como instrumento, um formulário, com perguntas abertas e fechadas, mediante assinatura do Termo de Consentimento Livre e Esclarecido. Após as entrevistas, ocorreu a aferição de medidas antropométricas (peso, altura), para cálculo do Índice de Massa Corporal (IMC). Em seguida, foram aferidas a pressão arterial e a glicemia casual dos caminhoneiros. Os autores compareceram ao local do estudo durante duas semanas, em dias alternados, no turno da manhã, totalizando dez coletas.

Os dados foram digitados e analisados com a utilização do software Statistical Package for the Social Science (SPSS) versão 17.0. Após a digitação, procedeu-se a análise dos dados. Foram calculadas estatísticas descritivas simples como frequências percentuais e médias, desvio padrão, valores máximos e 
mínimos. Para buscar os fatores de risco associados com a condição de portador de hiperglicemia, utilizouse o teste de $X^{2}$, com os respectivos valores de $\mathrm{p}$. A discussão foi feita à luz dos conhecimentos produzidos sobre o tema.

Os dados foram coletados mediante o consentimento expresso da população estudada, e aprovação do Comitê de Ética do Centro Universitário UNINOVAFAPI CAAE no 0047.0.043.000-10. Destaca-se que foram obedecidos todos os aspectos éticos contidos na Resolução 466/2012, que trata de ética envolvendo seres humanos.

\section{RESULTADOS}

A população estudada totalizou 74 caminhoneiros que trafegavam por Teresina. A Tabela 1 apresenta as características dos entrevistados quanto a procedência, idade, renda salarial, estado civil, escolaridade e tempo de profissão. Dentre o grupo analisado, prevaleceu o sexo masculino (100\%), a maioria era procedente da região Nordeste $(48,6 \%)$, seguida da região Sul $(25,6 \%)$, com faixa etária predominante de 46 a 74 anos $(59,5 \%)$ com amplitude de idade que variou de 25 a 74 anos. Em relação à renda salarial, pôde-se verificar que a maioria possuía renda de até seis salários mínimos $(77 \%)$. O estado civil mais frequente entre os caminhoneiros foi casado, com $66,2 \%$. Em relação ao grau de instrução, a expressiva maioria tinha estudo compatível com o ensino fundamental (77\%). Ao investigar o tempo de profissão, verificou-se que a maioria trabalhava há mais de 30 anos $(85,1 \%)$ (Tabela 1 ).

Tabela 1 - Dados sociodemográficos da população do estudo. Teresina, 2014.

\begin{tabular}{lcc}
\hline Variáveis & $\mathbf{N}$ & $\%$ \\
\hline Procedência & & \\
\hline Nordeste & 36 & 48,6 \\
Norte & 02 & 2,7 \\
Sul & 19 & 25,6 \\
Sudeste & 12 & 16,2 \\
Centro-Oeste & 05 & 6,7 \\
\hline Total & 74 & 100 \\
\hline Faixa etária (anos) & & \\
\hline 25 - 45 & 30 & 40,5 \\
$46-74$ & 44 & 59,5 \\
\hline Total & 74 & 100 \\
\hline Renda & & 77,0 \\
\hline Até 6 salários & 57 & 23,0 \\
6 salários e mais & 17 & 100 \\
\hline Total & 74 & 100,0 \\
\hline Sexo & & - \\
\hline Masculino & 74 & 100 \\
Feminino & - & \\
Total & 74 & 66,2 \\
\hline Estado civil & & 33,8 \\
Casado & 49 & 100 \\
Solteiro & 25 & 77,0 \\
\hline Total & 74 & 23,0 \\
\hline Escolaridade & & 100 \\
Até ensino fundamental & 57 & 14,9 \\
Ensino médio e superior & 17 & 100 \\
\hline Total & 74 & \\
\hline Tempo de profissão & 11 & \\
\hline Até 30 anos & 63 & \\
Acima de 30 anos & 74 & \\
\hline Total & & \\
\hline Fonte: Souza ATS, et al., & & \\
\hline
\end{tabular}


Quanto à distribuição dos caminhoneiros de acordo com a classificação dos níveis de pressão arterial (PA), 53 apresentaram níveis pressóricos dentro da faixa normal, representando $71,6 \%$ do total. Foram incluídos na faixa de hipertensão leve $15(20,2 \%)$ da população do estudo. Na categoria hipertensão moderada, foram incluídos $6(8,2 \%)$ dos indivíduos, totalizando assim, o percentual de $28,4 \%$ de indivíduos hipertensos (Tabela 2).

Tabela 2 - Distribuição dos níveis pressóricos da população do estudo. Teresina, 2014.

\begin{tabular}{lcc}
\hline & $\mathbf{N}$ & $\%$ \\
\hline Normotenso & 53 & 71,6 \\
Hipertensão leve & 15 & 20,2 \\
Hipertensão moderada & 6 & 8,2 \\
Hipertensão grave & - & - \\
\hline Total & 74 & 100 \\
\hline
\end{tabular}

Fonte: Souza ATS, et al., 2020.

Na população estudada, verificou-se que o IMC variou de 20 a 38, com média igual a $28,4(\mathrm{dp}=4,06)$, glicemia casual entre 60 a $490 \mathrm{mg} / \mathrm{dL}(\mathrm{dp}=86,44)$ e consumo alimentar diário variando entre 1 a 6 refeições/dia, com média de 2,6 ( $d p=1,29)$ (Tabela 3).

Tabela 3 - Média, mediana, moda, desvio padrão, mínimo e máximo do IMC, glicemia casual e número de refeições/dia na população do estudo. Teresina, 2014.

\begin{tabular}{lccc}
\hline & IMC & Glicemia casual & $\mathbf{N}^{\circ}$ de refeições/dia \\
\hline $\mathrm{N}$ & 74 & 74 & 74 \\
Média & 28,4 & 114,7 & 2,6 \\
Mediana & 29,0 & 88,0 & 3,0 \\
Moda & 26,0 & - & 3,0 \\
Desvio padrão & 4,065 & 86,445 & 1,292 \\
Mínimo & 20 & 60 & 1 \\
Máximo & 38 & 490 & 6
\end{tabular}

Fonte: Souza ATS, et al., 2020.

Verificou-se, que $75,7 \%$ da população estudada, encontrava-se com glicemia dentro da normalidade e $24,3 \%$ apresentavam hiperglicemia (>200 $\mathrm{mg} / \mathrm{dL}$ ), considerando-se a glicemia casual (Figura 1).

Figura 1 - Distribuição da população do estudo segundo os níveis glicêmicos. Teresina, 2014.

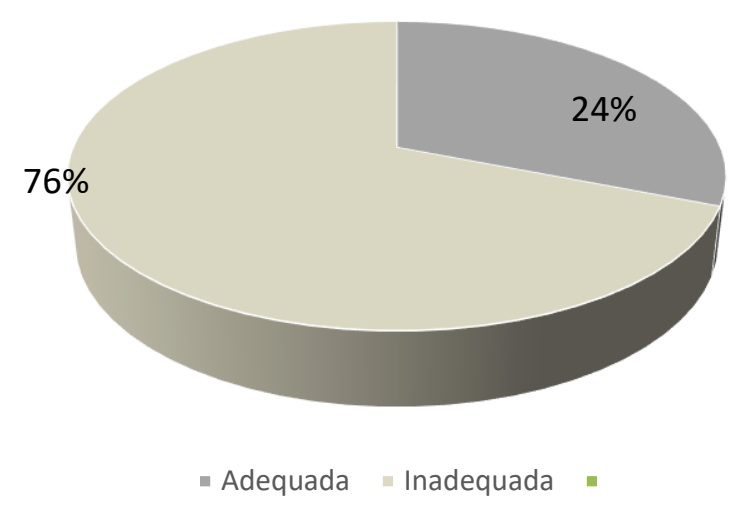

Fonte: Souza ATS, et al., 2020.

Observou-se que a maioria dos caminhoneiros (69\%) consomem uma alimentação inadequada, com predomínio de massas, doces, carnes vermelhas e gordas e refrigerantes. Apenas $31 \%$ referiram consumo 
alimentar dentro do padrão considerado adequado pelo estudo, com base em recomendações do Ministério da Saúde (Figura 2). O Ministério da Saúde, em âmbito nacional, recomenda o consumo de maiores quantidades de frutas, legumes e verduras e uma menor quantidade de gorduras, açúcares e sal.

Figura 2 - Distribuição da população do estudo quanto à adequação da alimentação. Teresina, 2014.

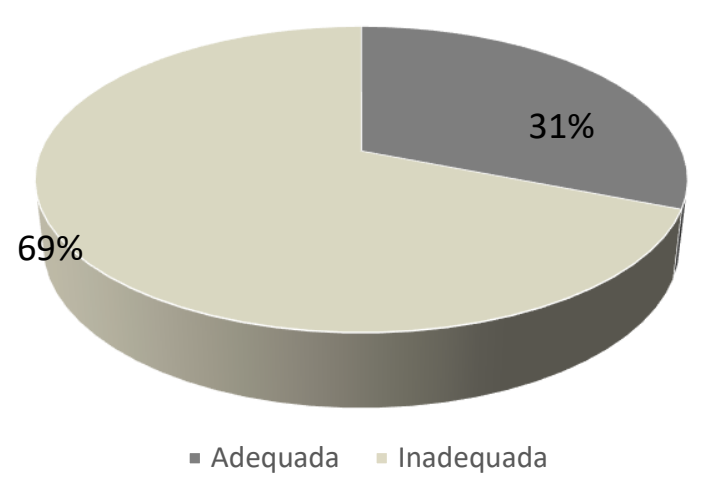

Fonte: Souza ATS, et al., 2020.

Ao investigar a associação da hiperglicemia com a dieta, foi possível verificar que a alimentação inadequada tem associação estaticamente significativa quando comparada à adequada $\left(x^{2}=7,235, p=\right.$ 0,007). Também foi associado o IMC de 26 a 38, quando comparado ao classificado entre 20 a $25\left(X^{2}=9,015\right.$, $p=0,003)$ e o nível pressórico acima do normal (hipertenso) $\left(X^{2}=17,156, p=0,00\right)$. Entretanto ter diabetes na família não mostrou significância estatística com o fato de 0 caminhoneiro apresentar hiperglicemia $\left(X^{2}=\right.$ 3,091, $p=0,07$ ) (Tabela 4).

Tabela 4 - Associação da glicemia casual com alimentação, IMC, diabetes na família e níveis pressóricos na população do estudo. Teresina, 2014.

\begin{tabular}{|c|c|c|c|c|c|c|}
\hline & \multicolumn{4}{|c|}{ Glicemia } & \multirow[b]{3}{*}{$\mathbf{N}$} & \multirow[b]{3}{*}{$\%$} \\
\hline & \multicolumn{2}{|c|}{ Normal } & \multicolumn{2}{|c|}{ Hiperglicemia } & & \\
\hline & $\mathbf{N}$ & $\%$ & $\mathbf{N}$ & $\%$ & & \\
\hline \multicolumn{7}{|l|}{ Alimentação } \\
\hline Adequada & 22 & 29,7 & 1 & 1,4 & 23 & 31,1 \\
\hline Inadequada & 34 & 45,9 & 17 & 23,0 & 51 & 68,9 \\
\hline Total & 56 & 75,7 & 18 & 24,3 & 74 & 100 \\
\hline Estatística: $\mathrm{X}^{2}=$ & 7,235 & $p=0,007$ & & & & \\
\hline \multicolumn{7}{|l|}{ IMC } \\
\hline 20 a 25 & 20 & 27,4 & 35 & 47,9 & 55 & 77,3 \\
\hline 26 a 38 & 0 & 0 & 19 & 24,7 & 19 & 22,7 \\
\hline Total & 20 & 27,4 & 54 & 72,6 & 74 & 100 \\
\hline Estatística: $\mathrm{x}^{2}=$ & 9,015 & $p=0,003$ & & & & \\
\hline \multicolumn{7}{|c|}{ Diabetes na família } \\
\hline Não & 32 & 43,2 & 06 & 8,1 & 38 & 51,4 \\
\hline Sim & 24 & 32,4 & 12 & 16,2 & 36 & 48,6 \\
\hline Total & 56 & 75,7 & 18 & 24,3 & 74 & 100 \\
\hline Estatística: $\mathrm{x}^{2}=$ & 3,091 & $p=0,07$ & & & & \\
\hline \multicolumn{7}{|c|}{ Níveis pressóricos } \\
\hline Hipertenso & 9 & 12,2 & 12 & 16,2 & 21 & 28,4 \\
\hline Normotenso & 47 & 63,5 & 6 & 8,1 & 53 & 71,6 \\
\hline Total & 56 & 75,7 & 18 & 24,3 & 74 & 100 \\
\hline Estatística: $\mathrm{x}^{2}=$ & 17,156 & $p=0,00$ & & & & \\
\hline
\end{tabular}

Fonte: Souza ATS, et al., 2020. 


\section{DISCUSSÃO}

Por muitas vezes, o DM2 apresenta-se silenciosa e o descontrole glicêmico pode ser o primeiro parâmetro a indicar um possível diagnóstico do diabetes, sendo um fator que deve ser melhor investigado. As alterações glicêmicas servem de grande alerta para os profissionais de saúde, principalmente ao enfermeiro, no acompanhamento das pessoas em risco de desenvolver DM2 e de intervenções que estimulem mudanças de hábitos, pois propicia aos usuários acesso ao conhecimento sobre seu processo saúde-doença, estimulando atitudes positivas no seu cuidado, retardando ou prevenindo o aparecimento do diabetes (ALENCAR DC, et al., 2019).

No que se refere ao fator sociodemográfico, todos os sujeitos do estudo eram do sexo masculino, a maioria provenientes da região Nordeste, idade entre 46 a 74 anos, casados, com renda de até seis salários mínimos, baixo nível de escolaridade, e com tempo de profissão acima de 30 anos.

Com relação à escolaridade, os achados foram semelhantes ao encontrado em estudo, que evidenciou baixa escolaridade de $68 \%$ dos caminhoneiros (ARAÚJO TME, et al., 2015). A baixa escolaridade é preocupante, pois é proporcional ao nível de conhecimento sobre prevenção dos fatores de riscos para 0 desenvolvimento das doenças, de modo geral, e especialmente do DM. A baixa escolaridade causa dificuldades, tanto ao acesso às informações, como a compreensão dos mecanismos que envolvem a hiperglicemia e seu tratamento, diminuindo as oportunidades de aprendizagem quanto aos cuidados com a saúde (SANTOS EKM, et al., 2018).

Nas últimas décadas, tem sido observado um aumento na prevalência de DM2. Tal fato tem sido atribuído às modificações do estilo de vida, ao aumento das taxas de sobrepeso e obesidade e ao envelhecimento populacional (MATOS EC, et al., 2018). Os achados do estudo evidenciaram que o IMC, apresentou média de $28,4 \mathrm{Kg} / \mathrm{m}^{2}$ e conforme a Organização Mundial de Saúde (OMS), valores de IMC $\geq$ a 25,0 e < 30,0 indicam adulto com sobrepeso (CALLO G, et al., 2016).

Muito se tem analisado e discutido sobre esta temática, mas parece haver consenso que os dois principais fatores relacionados com a elevação das prevalências de obesidade são mudanças nos hábitos alimentares e diminuição da atividade física (CAVALCANTE MLR, et al., 2016).

A obesidade e o sobrepeso afetam diretamente a saúde, levando os indivíduos a desenvolver doenças crônicas como hipertensão arterial, diabetes mellitus, acidente vascular encefálico (AVC), doenças cardíacas, hipercolesterolemia. A obesidade pode decorrer de vários fatores somatórios ou não somatórios que envolvem questões biológicas, históricas, ecológicas, econômicas, sociais, culturais e políticas. O aumento da massa corporal está diretamente ligado ao DM, permitindo compreender que o aumento de peso provoca resistência à insulina, levando ao desenvolvimento do DM2. Sendo assim, é de fundamental importância o controle de peso em pessoas diabéticas (LIMA LL, et al, 2015).

Apesar de o DM2 ser o tipo de diabetes mais comum, seu diagnóstico precoce não é frequente, uma vez que altos níveis de glicemia podem passar despercebidos e não apresentar sintomas evidentes (SMELTZER SC e BARE BG, 2015). Dados corroboram com outro estudo, em que foram encontrados caminhoneiros com glicemia capilar no valor de $472 \mathrm{mg} / \mathrm{dL}$, e estes não apresentavam sintomas aparentes de hiperglicemia (MAGALHÃES HJC, et al., 2015).

As complicações causadas pela evolução do DM, ou especificamente naqueles que perduram durante anos com alterações na tolerância à glicose, sem terem sidos diagnosticados e tratados, tornam clara a importância do prévio diagnóstico e a necessidade da implementação do tratamento correto, uma vez que a hiperglicemia é uma complicação da história natural desta doença (FUZINATO SF, et al., 2016).

$O$ fracionamento adequado das refeições representa um item importante na dieta da população em geral, pois facilita o controle glicêmico (BALDONI NR, 2017). No presente estudo, notou-se que os caminhoneiros têm em média, 2,6 refeições ao dia, uma alimentação inadequada, que relacionadas ao sobrepeso, são fatores propensos no desenvolvimento do DM2. No grupo pesquisado, apenas $31 \%$ referiu consumo alimentar dentro do padrão considerado adequado pelo Ministério da Saúde. 
Em relação aos hábitos alimentares adequados, o Ministério da Saúde, em âmbito nacional, recomenda o consumo de maiores quantidades de frutas, legumes e verduras e uma menor quantidade de gorduras, açúcares e sal, por terem implicações profundas na saúde e na qualidade de vida das pessoas. Recomendase, ainda, três porções de frutas por dia. Sugere-se fracionar o plano alimentar em seis refeições, sendo três principais e três lanches. Quanto à forma de preparo dos alimentos, deve-se dar preferência aos grelhados, assados, cozidos no vapor ou até mesmo crus (ARAÚJO ESS, et al., 2018).

Neste estudo, apenas 31,1\% relataram alimentação adequada, diferindo do estudo "Fatores de risco para obesidade entre caminhoneiros que trafegam na BR 381 ", em que $61,68 \%$ relataram preferir alimentos in natura e processados, $88,78 \%$ disseram fazer suas refeições em restaurantes, sendo que $77,5 \%$ não seguiam o horário habitual das refeições e, muitos destes, sequer as faziam por não disporem de tempo, alegando que não cumpririam o horário de chegada. Tudo isso implica hábitos alimentares inadequados, corroborando com os achados do estudo (GUEDES HM, et al., 2009).

A prática de atividade física de forma sistemática traz benefícios na musculatura esquelética, modificam o perfil lipídico, reduzem os níveis pressóricos e o risco de desenvolvimento de diabetes. Com relação a prática regular, entende-se que é muito difícil de ser aplicada aos caminhoneiros, em função de suas atividades laborais não terem uma regularidade quanto ao horário diário para iniciar ou terminar. No cotidiano de trabalho, ao longo do dia, permanecem por muitas horas nas estradas, longe dos centros urbanos (ARAÚJO TME, et al., 2015).

Segundo diretriz que trata da atividade física para adultos, é necessária a prática de atividade física de intensidade moderada, todos os dias da semana, para redução do risco de doenças crônicas, particularmente, hipertensão arterial e diabetes. No DM2, o excesso de peso e a obesidade estão fortemente associados ao desenvolvimento da doença, uma vez, que a obesidade é o determinante isolado mais relevante para a resistência à insulina. Exercícios físicos regulares ajudam a diminuir o peso corporal em obesos, do mesmo modo que agem reduzindo a necessidade do uso frequente dos hipoglicemiantes orais, diminuindo a resistência à insulina e contribuindo para um melhor controle glicêmico (MATOS EC, et al., 2018).

Estudo realizado em Campinas relata problemas de uso de álcool e drogas, sedentarismo, sobrepeso e hipertensão arterial como os principais problemas de saúde que acometem a categoria dos caminhoneiros (MASSON VA e MONTEIRO MI, 2010). Já em São Paulo, os resultados de pesquisa realizada não se assemelharam aos observados, onde dentre 258 motoristas de caminhão, verificou-se que $82 \%$ tinham IMC $>25 \mathrm{~kg} / \mathrm{m}^{2}$ e $7 \%$ glicemia $>110 \mathrm{mg} / \mathrm{dL}$ (CAVAGIONE LC, et al., 2008). Os resultados encontrados neste estudo foram de $22,7 \%$ de caminhoneiros com IMC>25 Kg/m² e $24,3 \%$ que apresentaram glicemia > 110 $\mathrm{mg} / \mathrm{dL}$.

Outra condição comum em pacientes com hiperglicemia é a hipertensão arterial, sendo o maior fator de risco para complicações cardiovasculares, como cardiopatia isquêmica, nefropatia e retinopatia. No DM2, a hipertensão está associada frequentemente como parte da síndrome metabólica. Os níveis de pressão arterial adequados e que devem ser atingidos se baseiam em ensaios clínicos que apontam benefício quando há redução da pressão sistólica em $<130 \mathrm{mmHg}$ e $<80 \mathrm{mmHg}$ em indivíduos com diabetes (ARAÚJO TME, et al., 2015). Os níveis pressóricos encontrados neste estudo foram de $71,6 \%$ normotensos, e com percentil de $28,4 \%$ de caminhoneiros hipertensos.

Quanto ao tempo de profissão, os resultados deste estudo corroboram com estudo realizado no Sul do Brasil, que mostrou uma jornada de trabalho diária longa, maior que 8 horas, tempo acima de 30 anos dedicado à ocupação, por conseguinte, comprometendo o tempo de sono e descanso, ocasionando problemas na saúde física e mental, podendo gerar problemas como fadiga, tensão câimbras e dores. O sono insuficiente e o cansaço podem predispor ao estresse emocional, depressão e sentimentos negativos, como angústia e ansiedade, os quais podem ter relação com distúrbios alimentares, repercutindo em obesidade, problemas digestivos e outros (QUEIROZ BO, et al., 2019).

Entender a Saúde do Homem como um conjunto de ações de promoção, prevenção, assistência e recuperação da saúde, disponíveis nos diferentes níveis de atenção se faz necessário, bem como priorizar a 
atenção básica, com foco na Estratégia Saúde da Família, porta de entrada do sistema de saúde integral, hierarquizado e regionalizado (ARAÚJO TME, et al., 2015).

A falta de procura pelos serviços de atenção primária faz que o indivíduo se prive da proteção necessária à preservação de sua saúde e passe a recorrer a procedimentos desnecessários. Muitos agravos poderiam ser evitados caso os homens adotassem, com frequência, as medidas de prevenção primária. O estudo contribuiu para mostrar o problema da falha na procura dos serviços de saúde pela categoria estudada e teve como limitação o fato de ter sido coletado em um local (posto de combustível) que não permitia melhor acolhimento pelos pesquisadores, o que provocou a recusa de muitos candidatos em participar.

Portanto, são necessários mais estudos com caminhoneiros, assim como deve ser mais abordada e debatida a Política Nacional de Atenção Integral à Saúde do Homem, essencial para maior aprofundamento sobre o tema. Entendemos que a implementação da Política de Atenção Integral à Saúde do Homem, com a organização de seus serviços de modo a ampliar seu acesso, favorecerá significativamente a saúde masculina. Os homens em geral, e, especialmente, os caminhoneiros, precisam ser incluídos em estratégias de educação em saúde, para serem alcançados pelas ações de atenção primária à saúde.

\section{CONCLUSÃO}

Os resultados do estudo evidenciaram que os caminhoneiros que trafegam pela capital do Piauí, embora não em sua maioria, mas em uma parcela considerável, apresentaram níveis glicêmicos elevados, sobrepeso e níveis pressóricos alterados, que se constituem fatores de risco predisponentes para o desenvolvimento do diabetes mellitus. O presente estudo, ao demonstrar a vulnerabilidade dos caminhoneiros que trafegam por Teresina, poderá possibilitar aos profissionais de saúde a construção de estratégias de prevenção e controle do diabetes nesta população, tendo em vista que tal categoria, em função do trabalho, fica à margem dos serviços públicos de saúde.

\section{REFERÊNCIAS}

1. ALENCAR DC, et al. Dimensões da qualidade de vida afetadas negativamente em pessoas vivendo com diabetes mellitus. Rev Pesq Cuid Fundam, 2019; 11(1): 199-204.

2. ARAÚJO ESS, et al. Cuidado de enfermagem ao paciente com diabetes fundamentado na Teoria de King. Rev Bras Enferm, 2018; 71(3): 1157-1163.

3. ARAÚJO TME, et al. Prevalência da hipertensão arterial sistólica entre caminhoneiros que trafegam pela cidade de Teresina. Sanare (Sobral), 2015; 14(1): 38-45.

4. BALDONI NR. Perfil nutricional de pacientes com diabetes mellitus tipo 2 em seguimento nas unidades de saúde da família. Dissertação (Mestrado em Ciências). Universidade de São Paulo, Ribeirão Preto, 2017; 119p.

5. BARROSO SV, BIAZON ACB. Influência da atividade física no tratamento da diabetes mellitus tipo 1 e tipo 2 . Rev Saúde e Biol, 2017; 12(1): 68-73.

6. CALLO G, et al. Excesso de peso/obesidade no ciclo da vida e composição corporal na idade adulta: coorte de nascimentos de Pelotas, Rio Grande do Sul, Brasil, 1982. Cad. Saúde Pública, 2016; 32(4): e00174014.

7. CAVAGIONE LC, et al. Metabolic Syndrome in professional truck drivers who work on Highway BR-116 within the area of São Paulo City - Régis Bittencourt. Arq Bras Endocrinol Metab, 2008; 52(6): 1015-1023.

8. CAVALCANTE MLR, et al. Alimentação saudável e atividade física como condicionantes de síndromes metabólicas em adolescentes. Sanare (Sobral), 2016; 15(2): 47-53.

9. DIAS ACM, et al. Doce cuidado: serviço de atendimento farmacêutico e nutricional a pacientes diabéticos em uma farmácia universitária. Rev Conexão UEPG, 2018; 14(1): 53-61.

10. DIAS SM, et al. Níveis de conhecimento de pacientes diabéticos sobre a Diabetes Mellitus tipo II. Rev Interdisciplinar, 2018; 11(3): 14-21.

11. FLOR LS, CAMPOS MR. Prevalência de diabetes mellitus e fatores associados na população adulta brasileira: evidências de um inquérito de base populacional. Rev Bras Epidemiol, 2017; 20(1): 16-29.

12. FONTES FLL, et al. Relevância da roda de conversa no Programa HIPERDIA: foco na alimentação saudável e atividade física. Rev Eletr Acervo Saúde, 2019; 23(Suppl): e394.

13. FUZINATO SF, et al. Alterações nutricionais e metabólicas em diabéticos: desafios ao hiperdia de uma estratégia de saúde da família. Rev Bras Promoç Saúde, 2016; 29(2): 268-277. 
14. GIROTTO E, et al. Comportamentos alimentares de risco à saúde e fatores associados entre motoristas de caminhão. Ciênc Saúde Colet, 2020; 25(3): 1011-1023.

15. GUEDES HM, et al. Fatores de risco para obesidade entre caminhoneiros que trafegam na BR 381. Rev Enferm Bras, 2009; 2(2): 345-358.

16. ISER BPM, et al. Prevalência de diabetes autorreferido no Brasil: resultados da Pesquisa Nacional de Saúde 2013. Epidemiol Serv Saúde, 2015; 24(2): 305-314.

17. LIMA LL, et al. Prevalência de sobrepeso e obesidade em diabéticos tipo 2 atendidos no ambulatório de Endocrinologia de um Hospital Universitário. Rev Soc Bras Clin Med, 2015; 13(4): 251-256.

18. LUÍZ RR. O tamanho da amostra em investigações epidemiológicas. In: Medronho RA, organizer. Epidemiologia. 2. ed. São Paulo: Atheneu; 2009. p. 180-200.

19. MAGALHÃES HJC, et al. Comportamentos de risco para a infecção pelos vírus da hepatite $B$ em caminhoneiros de longa distância em São Luís-MA. Revista de Investigação Biomédica, 2015; 7(1): 35-46.

20. MALHEIROS TM, et al. A importância de iniciativas de prevenção e promoção da saúde em diabetes mellitus. Rede de Cuidados em Saúde, 2017; 11(3): 1-5.

21. MASSON VA, MONTEIRO MI. Estilo de vida, aspectos de saúde e trabalho de motoristas de caminhão. Rev Bras Enferm, 2010; 63(4): 533-540.

22. MATOS EC, et al. O impacto de um projeto de intervenção nutricional e atividade física sobre fatores de risco cardiovascular em um grupo de mulheres obesas atendidas no ambulatório do Hospital Universitário Júlio Müller, Cuiabá-MT. RBONE. 2018; 12(72): 418-430.

23. QUEIROZ BO, et al. As consequências da restrição de sono sobre a qualidade de vida de caminhoneiros. Diálogos Interdisciplinares, 2019; 8(8): 49-57.

24. SANTOS EKM, et al. Autocuidado de usuários com diabetes mellitus: perfil sociodemográfico, clínico e terapêutico. Rev Pesq Cuid Fundam, 2018; 10(3): 720-728.

25. SEIXAS AMFF, et al. Adesão ao tratamento em crianças com diabetes tipo 1: insulinoterapia e apoio familiar. Rev SBPH, 2016; 19(2): 62-80.

26. SILVA LG, et al. Vínculos empregatícios, condições de trabalho e saúde entre motoristas de caminhão. Rev Psicol Organ Trab, 2016; 16(2): 153-165.

27. SMELTZER SC, BARE BG. Brunner \& Suddarth: Tratado de enfermagem médico-cirúrgica. 13 ed. Rio de Janeiro: Guanabara Koogan; 2015; 2256p. 